\title{
Mothers' Views About Lumbar Puncture for Their Children in a Maternity and Children's Hospital in Najran, Saudi Arabia
}

This article was published in the following Dove Press journal: Pediatric Health, Medicine and Therapeutics

\section{Khaled Sadeq Alshaibari' \\ Eman Redhwan Hasan ${ }^{2}$ \\ Mayasa Zaid Dammaj' \\ Iman Ali Sharaf Adeen ${ }^{3}$}

'Department of Pediatrics, College of Medicine, Najran University, Najran, Saudi Arabia; ${ }^{2}$ Emergency Department, Al-Zafer Hospital, Najran, Saudi Arabia ${ }^{3}$ Pediatric Behavior and Development, King Fahad Medical City, Riyadh, Saudi Arabia
Correspondence: Khaled Sadeq Alshaibari Email khalid22ye@gmail.com
Background: Refusal rates for offered pediatric lumbar puncture (LP) are high in many parts of the world, potentially hindering diagnosis and treatment for thousands of children. There is relatively little research about why such rates are so high. Understanding the formation and development of mothers' opinions about LP could help identify barriers to accessing this modality to improve diagnostic and treatment outcomes in children with neurological and systemic diseases.

Methods: We surveyed mothers of hospitalized children in the Maternity and Children's Hospital in Najran in November and December 2018. We queried their familiarity with LP and their decision to accept or refuse LP when it was offered. The team recorded demographic data on survey respondents, as well as their stated reasons for their LP decisions, and used chi-square tests to evaluate the correlation between patient or parent characteristics and the decision to accept or refuse LP.

Results: A total of 202 mothers responded to the survey, with a mean age of $30.7( \pm 6.9)$; nearly all women were stay-at-home mothers (93.1\%). Most (61.4\%) lived in urban settings. Four in 10 respondents $(40.6 \%)$ were not familiar with LP. A sizable minority of 89 mothers $(44.0 \%)$ refused LP for their children when offered, most commonly citing fear of paralysis $(39.3 \%)$ as the reason for refusal. Those who accepted LP were more likely to do so following physician advice than for any other reason $(\mathrm{p}<0.001)$.

Conclusion: Lumbar puncture refusal rates may be even higher than previously reported, and there is a pressing need to educate women on the diagnostic and therapeutic benefits of LP for their children. Maternal education from physicians may help improve acceptance rates for the procedure.

Keywords: decision-making, maternal attitudes, pediatrics, survey data

\section{Introduction}

Lumbar puncture (LP) has been part of the medical canon of procedures for over 100 years, most commonly for diagnosing and treating conditions of the central nervous system (CNS). ${ }^{1}$ It is the only method of obtaining cerebrospinal fluid (CSF) without neurosurgical intervention, and it is thus a diagnostic mainstay in children with meningitis, CNS tumors, fever of unknown origin, febrile seizures and other conditions. Despite its inherent risks, LP has an excellent safety record, with few contraindications and low complication rates when properly performed. Failure to perform LP in the presence of infectious meningitis frequently leads to the administration of empiric antibiotics in hospital settings, which can result in 
antibiotic resistance and prolonged hospital stays with non-targeted treatment. ${ }^{2,3}$ In environments where resources are limited, LP is often the only available diagnostic and therapeutic intervention for neurological disorders. $^{4}$

Yet parental refusal rates for offered pediatric LP are high, often exceeding 30\%. Though high-income countries worldwide tend to have lower LP refusal rates, in several Gulf countries refusal rates are high regardless of income level. Reports from Kuwait showed an $80 \%$ refusal rate, and two separate studies showed $44 \%$ refusal rates in the United Arab Emirates and Saudi Arabia; all three countries are considered high-income nations. ${ }^{5-8}$ Another report from the upper middle-income country of Iraq showed an LP refusal rate of $60.8 \%$, with particularly high rates of refusal for infants and neonates (the group most likely to require LP for diagnosis). ${ }^{8,9}$ Half of clinicians surveyed in several sub-Saharan African countries felt that LP refusal created a major impediment to clinical care. ${ }^{4}$

Despite the high rates of LP refusal and the resultant clinical impact, there is only a small body of research on parental attitudes toward LP. Accordingly, there is not much understanding of the factors involved in parental decisions about LP for their children. Associated factors for high refusal rates have been described in some of the aforementioned studies, but have not been universally established.

This study took place at Maternity and Children's Hospital, a specialized care center in the major city of Najran, Saudi Arabia. The Kingdom of Saudi Arabia is considered a male-dominant society, where fathers make most major family decisions; however, mothers here make a significant portion of household and childcare decisions and thus have influence on consent for pediatric health care matters. ${ }^{10}$ Many Saudis receive health care information from friends and relatives shared on social media rather than from medical sources, and women may be more likely to receive health care information this way than men. ${ }^{11}$ This practice can lead to spread of misinformation and severe disparities in knowledge of and familiarity with basic medical procedures like LP.

This study aimed to understand maternal levels of procedural familiarity with LP and the characteristics and attitudes of mothers regarding LP for their children. Understanding these factors may lead to better access to LP, and thus to improved diagnostic and treatment outcomes in children with neurological conditions or systemic illnesses.

\section{Methods}

\section{Sample and Setting}

This study was conducted from November 2018 to December 2018 at Maternity and Children's Hospital in Najran. The sample consisted of mothers who accompanied their children during inpatient hospitalization at Maternity and Children's Hospital in Najran, regardless of the cause of admission. This hospital is the primary referral hospital for obstetrics, gynecology and pediatrics in the region, and is thus most likely to accurately represent the demographic makeup of the region's mothers. ${ }^{12}$

The Maternity and Children's Hospital of Najran therefore provides an opportunity to survey an important crosssectional representation of maternal attitudes about LP. The city of Najran, the capital of Najran province, is a rapidly growing metropolis of 500,000 residents in the southwest sector of the upper-income country of Saudi Arabia. It is located less than 10 kilometers from the border with Yemen, a lower-income nation that has a major historical connection with the Najran region and exerts significant cultural influence there; as a result, Yemenis comprise a large minority of the population served by the hospital. The catchment area of the hospital includes the urban environment of Najran, as well as the rural desert and mountain herding areas surrounding the city.

Required sample size was calculated to be 196 subjects, using the standard same size equation, a margin of error selection of $5 \%$ and a confidence level of $95 \%$.

\section{Data Collection}

Mothers accompanying their hospitalized children were invited to an interview with a member of our team, who explained the purpose of the survey and obtained consent from the participants. Respondents answered questions orally and responses were recorded by team members. The survey and the interview were conducted in Arabic, which was the native language of all of the interviewees. The questionnaire took approximately 10 minutes to complete, on average.

\section{Participant Socio-Demographic Characteristics}

The study team collected demographic information about all participating mothers, including data on age, nationality, rural or urban living status, level of educational attainment, number of children, whether or not the respondent worked outside of the home and which category of employment they had if they did work outside of home 
(private-sector work, government-related, or other employment). These data were anonymized by use of a code number for each respondent.

\section{Familiarity with Lumbar Puncture}

Questionnaire recipients were asked if they were familiar with the LP procedure, and submitted binary "yes" or "no" answers. Respondents were queried about how they became familiar with LP - through friends, relatives, medical personnel, media or other sources - and whether or not medical professionals had ever previously requested lumbar puncture for any of their children.

\section{Decision and Reasoning}

Responding mothers were asked whether they would consent to or refuse LP if the procedure was medically indicated, and asked about their reasoning for their decision. Participants could select from prepared responses for either decision. If they stated that they would consent to LP, the mothers could cite the potential for diagnosis or therapy, or could state that they were following medical advice, or state their own reasons for consent. If the mothers stated that they would refuse LP, they could cite a fear of paralysis, fear of death, concern about pain, the advice of friends or relatives, religious reasons, a fear of unreliable physicians or state their own reasons for refusal. Respondents were free to select multiple reasons for their decision.

\section{Data Analysis}

All analyses were performed using version 25 of the Statistical Product and Service Solutions (SPSS) software suite. $^{13}$ The program was used to create frequency and percentage distributions for all variables. For categorical demographic variables, chi-squared tests $(\chi 2)$ were used to determine correlative relationships and responses. For analysis involving continuous variables (maternal age and number of children), simple linear regression was used. To identify the odds of selection of a given value within a variable, such as the source of familiarity with LP or the reason for the decision about $\mathrm{LP}$, odds ratio (OR) using a $95 \%$ confidence interval (CI) was the most appropriate test.

For variables which had multiple possible values but small $\mathrm{n}$, the values were collapsed into fewer choices. This was the case for nationality; since cases contained one each of the nationalities Palestinian, Syrian and Nigerian, the nationality variable was collapsed into possible values of "Saudi" and "non-Saudi". We also performed this action in analysis with number of children; though we used number of children as a continuous variable, we also collapsed the variable into "large family" (arbitrarily defined as eight or more children), "small family" (three or fewer children) and "midsize family" (more than three but fewer than eight children).

To account for the presence of categorical variables, the team used dummy coding, in which new binary variables are created out of each of the possible values of a categorical variable (except for the comparison group). ${ }^{14}$ For example, rather than use the categorical "level of education" as a single variable in the analysis, we used the possible values "illiterate", "primary" and "university or higher" as separate variables, assigning them either a 1 (yes) or 0 (no) to create binary values (note that we did not create a separate value for "secondary/diploma", which we used as the comparison group). Variables were otherwise assumed to follow a normal distribution.

We also performed regression analyses between variables to ensure that they did not contribute redundant information; however, none of the variables had a correlation value of $r$ greater than 0.75 . In regression models and chisquared tests, two-tailed $p$ values less than $5 \%$, written as $p<0.05$, were considered statistically significant.

This survey study was conducted in accordance with the Declaration of Helsinki and was exempted from review. Approval to conduct the study was granted by the Institutional Review Board of Najran.

\section{Results}

\section{Participant Socio-Demographic Characteristics}

Demographic data are summarized in Table 1. A total of 202 mothers were interviewed. Most of the respondents $(78.2 \%)$ were Saudi, with a sizable Yemeni minority $(20.2 \%)$. The remaining three respondents were of Nigerian, Palestinian and Syrian origin, respectively. The majority of respondents $(61.6 \%)$ lived in urban settings. About half of mothers $(52.0 \%)$ had completed an intermediate level of education or below, and about half $(48.0 \%)$ had a secondary level of education or above.

Mean age of mothers was 30.7 years, with a standard deviation (SD) of 6.9 years. The age range was from 18 to 55 years. More than 9 out of 10 respondents were stay-athome mothers; out of the $7 \%$ who worked outside of 
Table I Demographic Characteristics for Survey Respondents

\begin{tabular}{|c|c|}
\hline Continuous Variables & Mean (SD) \\
\hline Maternal age (in years) & $30.7( \pm 6.9)$ \\
\hline Number of children for each mother & $3.9( \pm 2.7)$ \\
\hline $\begin{array}{l}\text { Categorical Variables } \\
\text { Nationality }\end{array}$ & $\mathbf{N}(\%)$ \\
\hline Saudi & 158 (78.2\%) \\
\hline Yemeni & $4 \mid(20.3 \%)$ \\
\hline Other & $3(1.5 \%)$ \\
\hline Nigerian & I (0.5\%) \\
\hline Palestinian & I $(0.5 \%)$ \\
\hline Syrian & I (0.5\%) \\
\hline \multicolumn{2}{|l|}{ Residency } \\
\hline Urban & $124(61.4 \%)$ \\
\hline Rural & 78 (38.6\%) \\
\hline \multicolumn{2}{|l|}{ Occupational Status } \\
\hline Stay-at-home mother & I 88 (93.1\%) \\
\hline Work outside of home & 14 (6.9\%) \\
\hline Teacher & $5(2.5 \%)$ \\
\hline Doctor & $3(1.5 \%)$ \\
\hline Private sector & $3(1.5 \%)$ \\
\hline Government & $2(1.0 \%)$ \\
\hline University faculty & I (0.5\%) \\
\hline \multicolumn{2}{|l|}{ Education Level } \\
\hline Illiterate & $36(17.8 \%)$ \\
\hline Primary & $43(21.3 \%)$ \\
\hline Intermediate & $26(12.9 \%)$ \\
\hline Secondary & $60(29.7 \%)$ \\
\hline Diploma & $3(1.5 \%)$ \\
\hline Universit & $33(16.3 \%)$ \\
\hline Post-graduate & $\mathrm{I}(0.5 \%)$ \\
\hline Total number of respondents & 202 \\
\hline
\end{tabular}

home, a plurality of them were teachers. Doctors, government workers, university faculty and private sector workers comprised the rest of the employed population. Mean number of children for each mother was 3.9, with an SD of 2.7. The range for the number of children was 1 to 15 . More than $10 \%$ of respondents (21 mothers) had eight or more children.

Bivariate analysis showed that the number of children was highly correlated with maternal age $(r=0.68$, $p<0.001)$ and inversely correlated with level of education ( $\mathrm{r}=-0.45, p<0.001)$. Work outside of the home was correlated with higher education level $(\mathrm{r}=0.31$, $p<0.001)$. Number of children was not correlated with work outside of the home. Level of education was not correlated with nationality, and these variables were not correlated with living in a rural or urban area.

\section{Familiarity with Lumbar Puncture}

Four in ten respondents (40.6\%) were not familiar with LP as a procedure (see Figure 1). Out of the $59.4 \%$ who were familiar with LP, the most commonly stated source of this familiarity (38.1\%) was a previous request for LP by a medical professional for one of their children. Medical personnel $(33.9 \%)$, friends $(33.1 \%)$ and relatives $(22.9 \%)$ were other common sources of familiarity with LP (see Figure 2).

Having a large family size (eight or more children) was correlated with familiarity with LP $(\mathrm{r}=0.22, p=0.002)$. Similarly, higher maternal age was also correlated with LP familiarity $(\mathrm{r}=0.23, p=0.001)$. Conversely, having a relatively small family size was correlated with a lack of familiarity about LP ( $\mathrm{r}=0.17, p=0.017)$. Familiarity with LP was not correlated with education level, nationality or occupational status (see Table 2).

Approximately $22.3 \%$ of respondents had a physician previously request an LP for a child in their household; this population had a higher likelihood of being familiar with the procedure $(\mathrm{OR}=2.11,95 \% \mathrm{CI} 1.79-2.49)$ than those who had not had a previous request.

\section{Decision and Reasoning}

Out of the 202 responding mothers, 89 (44.0\%) indicated that they would refuse LP for their child, and $113(56.0 \%)$ indicated that they would consent (see Figure 3 ). The most commonly stated reason for refusal was fear of paralysis (39.1\% of respondents who refused). The most commonly stated reason for consent, by far, was to follow physician advice $(67.3 \%$ of respondents who consented-see Table 3).

\section{Characteristics Associated with Decision and Reasoning}

Consent for LP was not statistically more or less likely for any socio-demographic variable, though mothers with eight or more children had a $71.4 \%$ acceptance rate. Consent was no more or less likely to be given when mothers had a previous request for LP for a child. Similarly, familiarity with LP did not increase the likelihood of consent. 
Prior familiarity with lumbar puncture

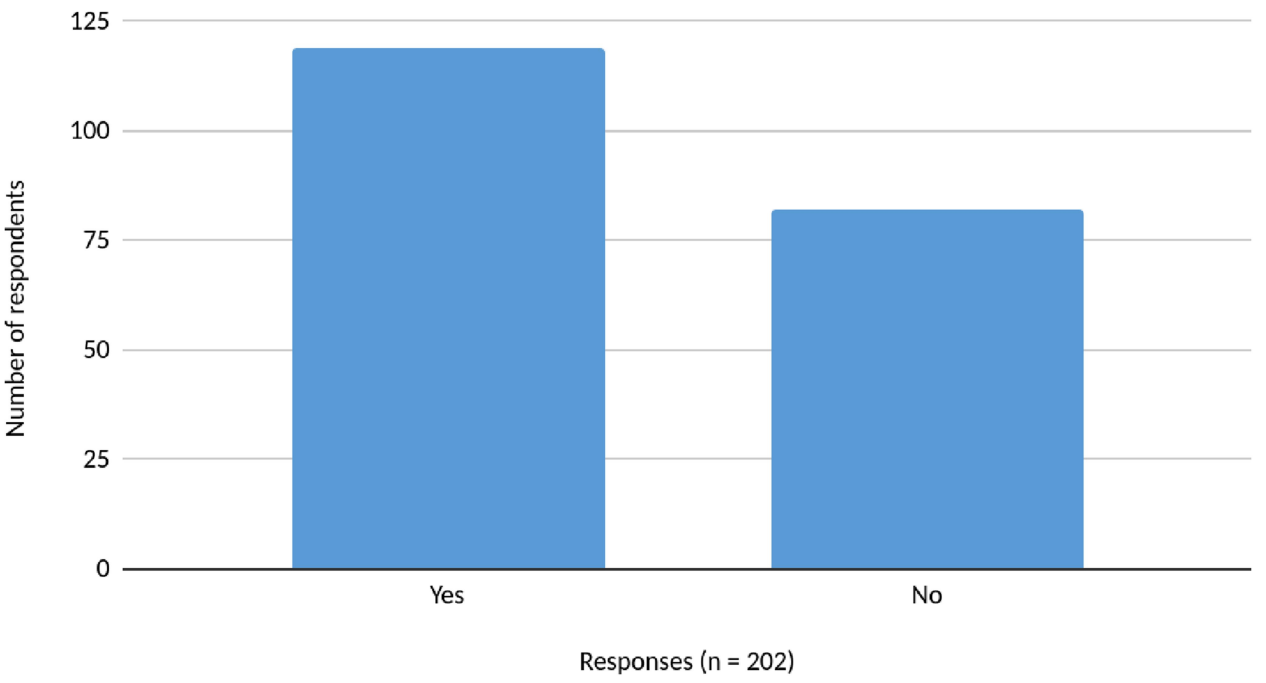

Figure I Respondents' familiarity with lumbar puncture.

\section{Source of Familiarity with Lumbar Puncture}

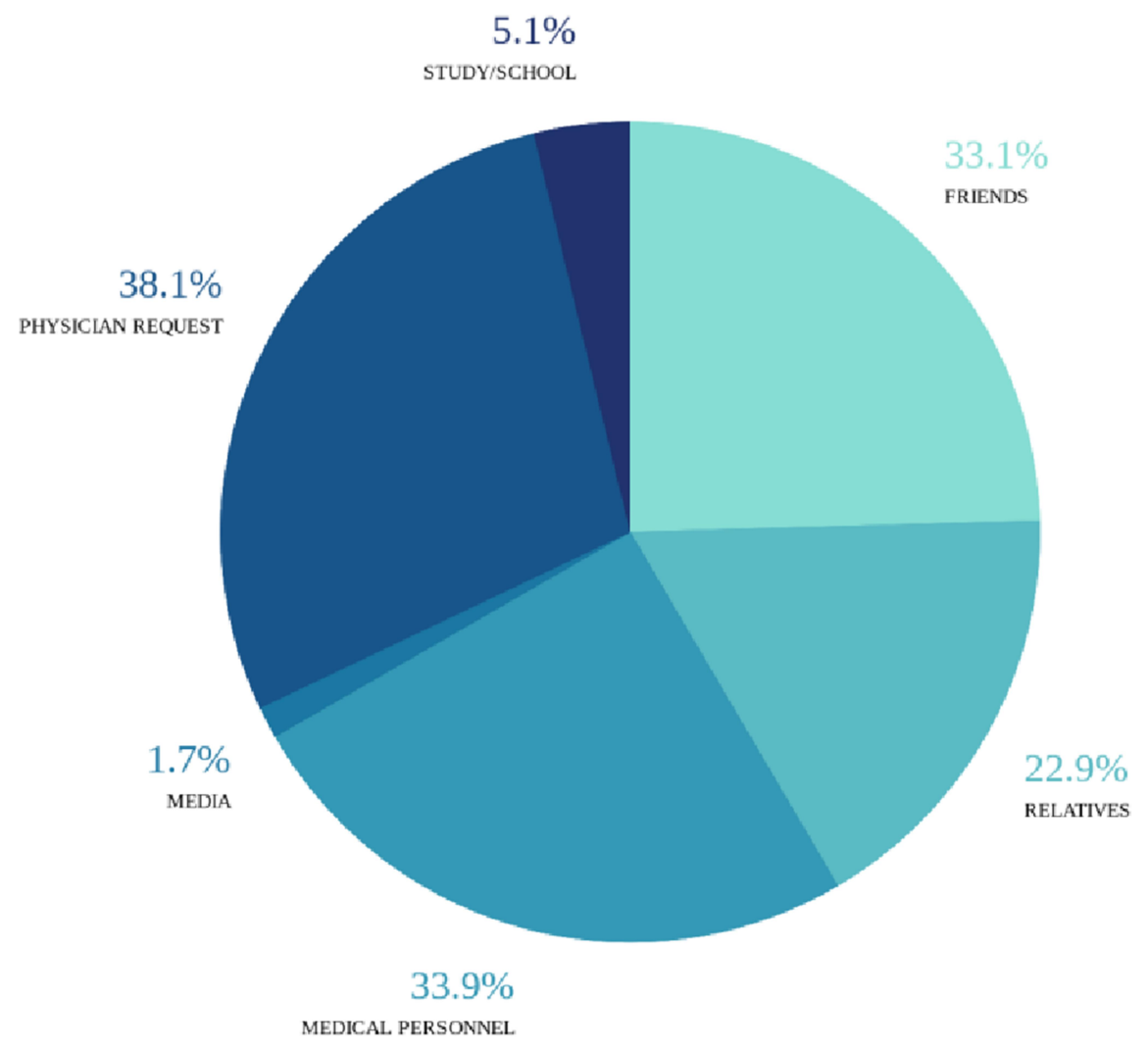

Figure 2 Source of respondents' familiarity with lumbar puncture. 
Table 2 Relationships Between Socio-Demographics and Familiarity with Lumbar Puncture

\begin{tabular}{|l|c|c|}
\hline Variables & $\begin{array}{c}\text { Pearson's } \\
\text { Coefficient }(\mathbf{r})\end{array}$ & $\begin{array}{c}\mathbf{p} \text {-value ( } \leq \mathbf{0 . 0 5} \text { in } \\
\text { bold) }\end{array}$ \\
\hline Higher maternal age & 0.23 & $\mathbf{0 . 0 0 1}$ \\
\hline Non-Saudi nationality & 0.12 & 0.087 \\
\hline Rural living & -.01 & 0.863 \\
\hline $\begin{array}{l}\text { Large family size }(\geq 8 \\
\text { children) }\end{array}$ & 0.22 & $\mathbf{0 . 0 0 2}$ \\
\hline $\begin{array}{l}\text { Small family size }(\leq 3 \\
\text { children) }\end{array}$ & -.17 & $\mathbf{0 . 0 1 7}$ \\
\hline High education level & -.04 & 0.554 \\
\hline Work outside of home & 0.03 & 0.690 \\
\hline
\end{tabular}

Some of the sources of familiarity, however, did show a relationship to the decision to consent to or refuse LP. Refusal was less likely when respondents heard about LP from a media source like the Internet, an article or television ( $\mathrm{OR}=0.53,95 \%$ CI $0.44-0.63)$, or from a study (OR $=0.52,95 \%$ CI $0.42-0.61)$.

Mothers were more likely to be following physician advice when giving consent (OR 3.05, 95\% CI 2.34-3.98, $p<0.001)$. They were also more likely to cite fear of paralysis as the reason for refusing consent (OR 2.17, 95\% CI 1.73-2.72); they were even more likely to have a fear of paralysis if they had previously been familiar with LP (OR 3.38, 95\% CI 1.57-7.26). Rural residents were less likely to have a fear of paralysis about LP (OR $0.92,95 \%$ CI $0.86-0.99)$.

\section{Discussion}

\section{Consistency with Prior Data}

Saudi Arabia is a country where men are typically heads of households, and thus generally considered the primary household decision makers, with women assuming a larger role in in-home matters and either indirectly or directly impacting the decision-making process. However, a previous study by Alwahbi and colleagues, which surveyed families of 750 children with meningitis symptoms in four Saudi hospitals, showed that fathers and mothers jointly made the decision in $66 \%$ of the cases where LP was refused. ${ }^{7}$ The overall refusal rate for LP in that study was $44.3 \%$; our study (which used survey data from mothers exclusively) was very consistent with that prior result, with a refusal rate of $44.0 \%$. A previous smaller study by Narchi and colleagues from a hospital in the United Arab Emirates involving 55 families also showed a pediatric LP refusal rate of $44 \%{ }^{6}$

This remarkable level of consistency generated several observations. First, the baseline probability of refusal for an offered LP appears to be higher than previously imagined. Refusal rates of $24 \%$ in places like Malaysia had been considered high, especially in comparison to Western countries; that rate would be a drastic reduction from the results in several Gulf countries. ${ }^{3,6,7}$ Second, it appears

\section{Maternal Acceptance or Refusal for Lumbar Puncture}

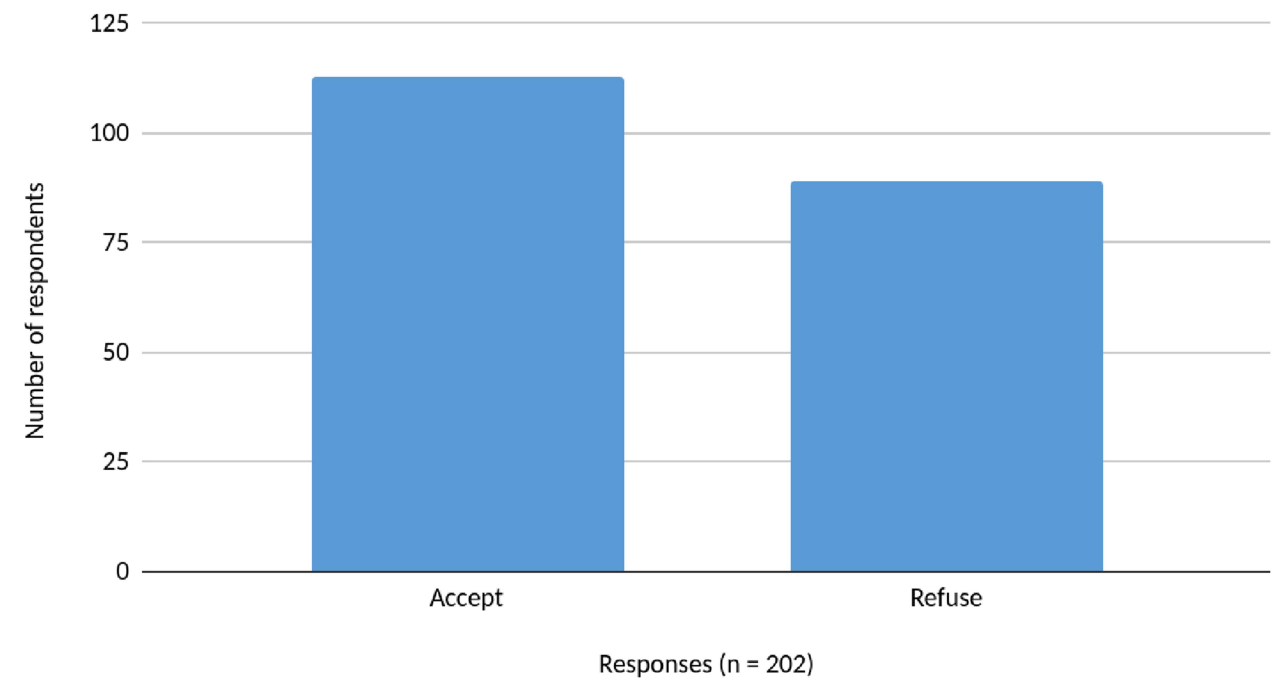

Figure 3 Acceptance (consent) and refusal for lumbar puncture for responding mothers. 
Table 3 Commonly Cited Reasons for Consenting to or Refusing Lumbar Puncture

\begin{tabular}{|l|l|}
\hline \multicolumn{2}{|c|}{ Cited Reasons for Lumbar Puncture Decision } \\
\hline Accept & Refuse \\
\hline Following physician's advice (67.3\%) & Fear of paralysis (53.9\%) \\
\hline Potentially diagnostic (24.8\%) & Injection site danger (18.0\%) \\
\hline Potentially therapeutic (20.4\%) & Fear of death (16.9\%) \\
\hline
\end{tabular}

Note: Respondents could select more than one variable, so percentages do not add to 100 .

that parent gender was not an important determinant of the likelihood of refusing an offered LP, as rates of refusal were the same no matter which parent makes the decision. Third, the reasons for the refusal show similar amounts of widespread prevalence between studies, and typically involve the fear of serious side effects or poor outcome, including death. Fourth, the prevalence of these fearsmore than $25 \%$ of families who refused LP cited fear of paralysis and/or death in each of these studies - is disproportionate to the rate of LP complications. ${ }^{16}$

\section{Specific Impact on Saudi Arabia}

Saudi families tend to be larger than many other countries of similar income or demographic, even in comparison to Saudi Arabia's smaller Gulf neighbors; it is statistically one of the youngest countries in the world, with over $50 \%$ of its population under the age of $25 .{ }^{17}$ Therefore, the absolute volume of pediatric neurological disease in Saudi Arabia, even at normal incidence rates, is high. The medical consequences of high LP refusal rates in the presence of pediatric neurological disease have been previously described: increased use of empiric intravenous antibiotics, increased use of resources, longer hospital stays, antibiotic resistance, delayed meningitis prophylaxis and higher risk of nosocomial infections and iatrogenic events. $2,3,18-20$

Two recent studies on parents' views on pediatric LP in other Saudi Arabian cities have helped provide important insights on the demographic, educational and sociological factors associated with these high refusal rates, and the studies have helped provide useful comparison of attitudes about LP between regions of the country. In a study of over 1200 parents conducted in Riyadh, the capital and largest city of the Kingdom, Muammar and colleagues demonstrated a direct association between low levels of educational achievement, lack of knowledge about LP and negative views about LP. Notably, they did not find correlation of negative views about LP with the gender of the parent. $^{21}$ Meanwhile, Almatawah and colleagues found their study of 466 parents to demonstrate relatively low rates of refusal $(12 \%)$ in the region of $\mathrm{Al}$-Ahsa, in eastern Saudi Arabia. Further studies may help delineate the correlations within the country regarding attitudes and refusal rates. $^{22}$

\section{Impact of Demographics}

Even though prior studies have described similar results, one of the more surprising aspects of this analysis was the apparent lack of correlation of maternal demographic factors with consent to or refusal of LP. In this study, the decision to accept or decline LP had no statistically significant correlations with maternal age, nationality, number of children, occupational status, or educational level. This phenomenon may be explained, in part, by the relatively close rates of acceptance $(56.0 \%)$ and refusal $(44.0 \%)$; it may be easier to establish correlations between variables when measured values tend toward extremes. ${ }^{23}$ For example, the 21 mothers who had eight or more children consented $71.4 \%$ of the time, and this population was the demographic correlate closest to statistical significance. Pearson's r, used here, has limitations with higher n, and Spearman's rank coefficient may have been more appropriate in some circumstances.

\section{Impact of Source of LP Familiarity}

On the other hand, source of familiarity with lumbar puncture appeared to be an important correlate of the decision to accept or refuse LP. When mothers learned about LP through media like books or the Internet, or by hearing about a clinical study, they were more likely to consent to the procedure. Though the $n$ for this subset of respondents is small, this finding is also consistent with prior studies, and may suggest potential benefits to increasing public awareness of the importance and safety of LP.

\section{Impact of Physicians on Consent}

Mothers were three times more likely to consent for LP for children following the advice of a physician than to consent for any other reason. Over $67.3 \%$ of women who consented to LP responded that they were following physician advice. These findings suggest that physicians remain the most important source of information about LP, and that families can use physician counsel as their 
primary (if not always exclusive) guides when deciding on LP for a child.

Alduraywish and colleagues showed that in Saudi Arabia, physicians are the primary source of medical information for $87.6 \%$ of survey respondents, despite the assumed competition from social media. Doctors are trusted by nearly $80 \%$ of the population; these percentages remain high despite readily accessible medical information online, which may or may not have been verified. Such a level of perceived trustworthiness about physicians provides two insights. First, it underscores the need for physicians to widely disseminate uniform, clear information about the safety of LP. Second, it suggests that patients are likely taking their cues from their physician about consent decisions, especially when the patient's knowledge base is limited. For example, out of the 49 respondents who consented to LP despite no previous familiarity with it, 30 of them $(61.2 \%)$ did so because they were following physician advice. Thus, the quality of physician presentation, tone, body language and content of information about LP to parents is of paramount importance.

Improving access to lumbar puncture in children with neurologic symptoms is of critical importance, and an understanding of the factors that influence maternal decisions about LP is of great value. Refusal of pediatric lumbar puncture creates a clinical and public health dilemma, and that will likely remain true as long as LP is the only non-surgical method of access to CSF. Entrenched anxieties about paralysis and death from LP remain, despite low rates of procedural complications. However, this study provides some promising evidence that targeted exposure to LP might help increase acceptance rates for the procedure. Clinicians will need to give careful explanations about the importance of LP to parents. Multidisciplinary approaches may be of great benefit, as nurses can help answer questions, allay worries, and provide a sense of what to expect during LP. Accurate and reassuring information from individual physicians, nurses and from public health organizations may help reduce these anxieties in the public sphere, and may be able to provide more children with the opportunity for effective treatment of neurological and systemic disease.

\section{Strengths and Limitations}

The design of this study had methodological strengths and limitations. The study was a single-site survey which used convenience sampling; however, the respondents appeared to be well representative of the population of mothers in Najran province. Levels of education, nationality, mean number of children and rural/urban population proportions in survey respondents closely matched data from the 2016 general demographic survey for Najran province from the Kingdom's General Authority for Statistics. ${ }^{15}$ A limitation of the study is that it queried mothers of children for allcause admissions, rather than specific admissions for a neurological problem or symptoms for which LP would be indicated. It is certainly possible that in the emergent presence of neurological disease, LP refusal rates would decrease.

\section{Conclusions}

Careful examination of the data revealed that pediatric lumbar puncture refusal rates among mothers in Najran remain high enough to pose a threat to public health. However, exposure to information about LP from sources these mothers trust-physicians, study data, and Internet sources-are correlated with decreased refusal rates. These associations hold regardless of education level, family size, or even previous experience with LP. This study provides important insight about how these correlations can be leveraged to deliver more targeted education to mothers, particularly with a multidisciplinary approach, about this safe and potentially life-saving procedure.

\section{Author Contributions}

All authors made substantial contributions to conception and design, acquisition of data, or analysis and interpretation of data; took part in drafting the article or revising it critically for important intellectual content; agreed to submit to the current journal; gave final approval of the version to be published; and agree to be accountable for all aspects of the work.

\section{Disclosure}

The authors report no potential conflicts of interest for this work.

\section{References}

1. Wynter WE. Four cases of tubercular meningitis in which paracentesis of the theca vertebralis was performed for the relief of fluid pressure. Lancet. 1891;1:981-982. doi:10.1016/S0140-6736(02)16784-5

2. Ling SG, Boey CC. Lumbar puncture refusal in febrile convulsion. Singapore Med J. 2000;41(10):485-488.

3. Deng CT, Zulkifl I HI, Azizi BH. Parents' views of lumbar puncture in children with febrile seizures. Med J Malaysia. 1994;49(3):263-268. 
4. Thakur KT, Mateyo K, Hachaambwa L, et al. Lumbar puncture refusal in sub-Saharan Africa: a call for further understanding and intervention. Neurology. 2015;84(19):1988-1990. doi:10.1212/ WNL.0000000000001561

5. Farag E, Husain E, Fathy H, Shawky A. Perceptions and attitude towards lumbar puncture (LP) among parents in Kuwait. KMJ 2009;41:307-310.

6. Narchi H, Ghatasheh G, Hassani NA, et al. Comparison of underlying factors behind parental refusal or consent for lumbar puncture. World J Pediatr. 2013;9:336-341. doi:10.1007/s12519-013-0419-z

7. Alwahbi ZM, Alzahrani AA, Alqhtani MM, Asiri WI, Assiri MA. Evaluation of Saudi Arabian parents' attitude towards lumbar puncture in their children for diagnosis of meningitis. Egypt J Hosp Med. 2018;70:1582. doi:10.12816/0044687

8. World Bank. World bank open data: free and open access to global developmental data. Available from: https://data.worldbank.org/.

9. Al-Hajjiah NN, Al-Shamsi MM, Al-Shami MM. The rate of parental refusal lumbar puncture in the Maternity and Children Teaching Hospital in Diwaniyah, Iraq. $J$ Pharm Sci Res. 2018;10 (10):2680-2681.

10. Almosaed N. Money and power in Saudi family. JKAU: Arts Humanities. 2008;16(2):61-87.

11. Alduraywish SA, Altamimi LA, Aldhuwayhi RA, et al. Sources of health information and their impacts on medical knowledge perception among the Saudi Arabian population: cross-sectional study. J Med Internet Res. 2020;22(3):e14414. doi:10.2196/14414

12. Kingdom of Saudi Arabia ministry of health. Available from: https:// www.moh.gov.sa/en/Ministry/MediaCenter/News/Pages/News-201912-31-001.aspx.

13. IBM Corporation. IBM SPSS Statistics for Windows, Version 25.0. Armonk, NY, USA: IBM Corp; 2017.
14. Alkharusi H. Categorical variables in regression analysis: a comparison of dummy and effect coding. Int J Educ. 2012;4 (2):202. doi:10.5296/ije.v4i2.1962

15. Kingdom of Saudi Arabia General Authority for Statistics. Demography survey data. Available from: https://www.stats.gov.sa/ sites/default/files/en-demographic-research-2016_2.pdf.

16. Ruff RL, Dougherty JH Jr. Complications of lumbar puncture followed by anticoagulation. Stroke. 1981;12(6):879. doi:10.1161/01. STR.12.6.879

17. Kingdom of Saudi Arabia general authority for statistics. Available from: https://www.stats.gov.sa/en/indicators/10.

18. Kimia A, Ben-Joseph EP, Rudloe T, et al. Yield of lumbar puncture among children who present with their first complex febrile seizure. Pediatrics. 2010;126(1):62-69. doi:10.1542/peds.2009-2741

19. Carroll W, Brookfield D. Lumbar puncture following febrile convulsion. Arch Dis Child. 2002;87(3):238-240. doi:10.1136/ adc.87.3.238

20. Broome ME, Bates TA, Lillis PP, McGahee TW. Children's medical fears, coping behaviour patterns and pain perceptions during a lumbar puncture. Eur J Cancer Care. 1994;3(1):31-38. doi:10.1111/j.13652354.1994.tb00007.x

21. Muammar NB, Al Rohaimi N, Aleid B, Al Harbi A, Yousif A. Level of awareness of parents toward pediatric lumbar punctures in Riyadh, Saudi Arabia. Saudi J Emerg Med. 2020;1(2):96-102. doi:10.24911/ SJEMed/72-1586249695

22. Almatawah Y, Busaleh F, Alkhars A, et al. (2020). Knowledge and attitude of parents toward pediatric lumbar puncture and its relationship with demographical factors in Al-Ahsa, Saudi Arabia. Med Sci. 2020;24(105):2883-2892.

23. Mukaka MM. Statistics corner: a guide to appropriate use of correlation coefficient in medical research. Malawi Med J. 2012;24(3):69-71.
Pediatric Health, Medicine and Therapeutics

\section{Publish your work in this journal}

Pediatric Health, Medicine and Therapeutics is an international, peerreviewed, open access journal publishing original research, reports, editorials, reviews and commentaries. All aspects of health maintenance, preventative measures and disease treatment interventions are addressed within the journal. Practitioners from all disciplines are invited to submit their work as well as healthcare researchers and patient support groups. The manuscript management system is completely online and includes a very quick and fair peer-review system. Visit http://www.dovepress.com/testimonials.php to read real quotes from published authors. 